\title{
Attosecond Time-Resolved Photoelectron Dispersion and Photoemission Time Delays
}

\author{
Q. Liao and U. Thumm \\ Department of Physics, Kansas State University, Manhattan, Kansas 66506, USA
}

(Received 22 May 2013; published 14 January 2014)

\begin{abstract}
We compute spectrograms and relative time delays for laser-assisted photoemission by single attosecond extreme ultraviolet pulses from valence band (VB) and $2 p$ core levels (CLs) of a $\operatorname{Mg}(0001)$ surface within a quantum-mechanical model. Comparing the time-dependent dispersion of photoelectron (PE) wave packets for VB and CL emission, we find striking differences in their dependence on the (i) electron mean free path (MFP) in the solid, (ii) screening of the streaking laser field, and (iii) chirp of the attosecond pulse. The relative photoemission delay between VB and $2 p$ PEs is shown to be sensitive to the electron MFP and screening of the streaking laser field inside the solid. Our model is able to reproduce a recent attosecond-streaking experiment [S. Neppl et al., Phys. Rev. Lett. 109, 087401 (2012)], which reveals no relative streaking time delay between $\mathrm{VB}$ and $2 p$ PEs.
\end{abstract}

DOI: 10.1103/PhysRevLett.112.023602

PACS numbers: 42.50.Hz, 42.65.Re, 79.60.-i

During the past decade, the combination of either an isolated attosecond extreme ultraviolet (XUV) pulse [1,2] or a train of attosecond XUV pulses [3] with a synchronized delayed femtosecond (fs) near-infrared (NIR) laser pulse has allowed the measurement of apparent photoemission time delays in the laser-assisted XUV photoionization of atoms in the gas phase $[2,3]$ and on solid surfaces $[4,5]$. Apparent relative photoemission delays are of the order of tens of attoseconds ( 1 as $=10^{-18} \mathrm{~s}$ ) $[2,4]$ or less [5] and yield information on the (i) primary XUV photoabsorption process, (ii) influence of the NIR-laser field, (iii) residual charge of the target [6], (iv) electronic correlation [7], and (v) collective (plasmonic) excitation processes on the PE propagation [8]. The temporal and spectral structure of both, electron wave packets and attosecond XUV pulses can be characterized based on streaked PE spectra $[9,10]$. NIR-laser-streaked XUV photoemission thus holds promise to enable the scrutiny of electronic dynamics in atoms and solids with unprecedented time resolution at the natural time scale of the electronic motion in matter [11,12].

Compared to gaseous atomic targets, the modeling of photoemission from solids is more challenging due to a complex electronic band structure [4,13,14], elastic and inelastic collisions inside the substrate [15-17], the screening of the NIR streaking field inside solids [16,17], and surface and bulk plasmon excitations $[8,18]$. In a first, proof-of-principles experiment on solids, a relative photoemission delay of $\Delta \tau_{\mathrm{VB}-4 f}=110 \pm 70$ as between PEs emitted from the valence band (VB) and $4 f$ core levels (CLs) of a W(110) surface was deduced from streaked PE spectra [4], initiating comprehensive theoretical studies [13-17,19,20]. Employing classical transport theory, the $4 f$ PEs were traced to be slower, originate in deeper layers than VB PEs, and thus need more time to escape through the surface $[4,15]$. Based on quantum-mechanical calculations, the measured relative time delay $\Delta \tau_{\mathrm{VB}-4 f}$ was interpreted as a result of the different wave function localization of the initial delocalized VB and localized CL states $[16,17]$.

A simple quantum-mechanical model [21], representing both CLs and VB levels in terms of lattice-centered, attractive Gaussian-shaped potentials of adjustable widths, is in accord with the large measured [4] delay $\Delta \tau_{\mathrm{VB}-4 f}$ for $\mathrm{W}(110)$. This may suggest that the relative streaking time delay $\Delta \tau_{\mathrm{VB}-4 f}$ is primarily determined by differences in the core- and VB-level wave function localizations. However, the agreement with the measured relative delay for $\mathrm{W}(110)$ appears to be serendipitous, as the simple model [21] does not reproduce the much smaller relative delay $\Delta \tau_{\mathrm{VB}-2 p}=$ $5 \pm 20$ as between $\mathrm{VB}$ and $2 p \mathrm{CL}$ emission extracted recently from measured streaked $\mathrm{PE}$ yields on a $\mathrm{Mg}$ (0001) surface [5]. This finding is surprising in so far as $\mathrm{Mg}$ is better approximated as a free-electron metal than $\mathrm{W}$, with a corresponding larger difference between VB and CL wave function localization. The simultaneous detection of VB and CL PEs was also analyzed within a heuristic model [5], assuming (i) the streaking field is strongly damped at the surface atomic layer [22], (ii) free PE propagation to the $\mathrm{Mg}(0001)$ surface, and (iii) PE escape depths equal to the PE-energy-dependent mean free path (MFP). Under these assumptions, the vanishing relative delay $\Delta \tau_{\mathrm{VB}-2 p}$ was traced to identical propagation times of VB and CL PEs to the surface, in sharp contrast to the classical interpretation of streaked photoemission from W(110) [4]. Solving the time-dependent Schrödinger equation for 1-dimensional model potentials, Borisov et al. [23] explained the vanishing delay $\Delta \tau_{\mathrm{VB}-2 p}$ as a result of photoemission from the $\mathrm{Mg}$ VB via dominant resonant interband transitions. While recent work on attosecond time-resolved photoemission focussed on relative time delays, obtained by spectrally averaging over PE streaking traces, streaked PE spectra also contain information on PE propagation and dispersion that is lost in the spectral average. This includes the 
influence of duration and chirp of the XUV pulse [9,10], skin depth of the streaking field, and substrate band structure on the PE wave packet.

We analyze streaked XUV PE yields from VB and $2 p$ CLs of $\mathrm{Mg}(0001)$ surfaces and their relative photoemission time delay for PE emission in direction perpendicular to the surface $(z$ axis). We model the free-electron-like $\mathrm{Mg}$ VB based on the "Chulkov potential" $U_{C}(z)$, representing the substrate band structure at the level of density-functional theory [24]. This effective potential reproduces the measured projected bandwidth, band gap, and surface-state (SS) energy of the $\operatorname{Mg}(0001)$ surface. The substrate in Neppl's experiment [5] consists of $a>30$ layer Mg film that was epitaxially grown on a $\mathrm{W}(110)$ surface and is assumed to have the same electronic properties as a $\operatorname{Mg}(0001)$ single crystal [25]. Here, we consider a slab of $31 \mathrm{Mg}$ atoms separated by the $\operatorname{Mg}(0001)$ lattice constant $a_{s}=2.6 \AA$. We use atomic units throughout this Letter unless stated otherwise. The highly delocalized VB states are obtained by diagonalizing $U_{C}(z)$ [Fig. 1(a)]. Occupied states cover a bandwidth of $\sim 7 \mathrm{eV}$ below the Fermi level. The SS is located inside the band gap at an energy of $-1.53 \mathrm{eV}$ (relative to the Fermi level). The highest eight VB states below the Fermi level are energetically very close to those found in the experiment [25].

To represent the completely localized $2 p$ CL electrons we combine the Yukawa potential $U_{Y}(z)=$ $\sum_{j}-e^{-\left|z-z_{j}\right| / \xi} / \sqrt{\left(z-z_{j}\right)^{2}+a_{0}^{2}}$, where $z_{j}=(16-j) a_{s}$ $(j=1,31)$ is the position of the $j$ th atom, with $U_{C}(z)$ to obtain the effective CL potential $U_{\mathrm{CL}}=U_{C}(z)+U_{Y}(z)$ [Fig. 1(a)]. By adjusting the screening length $\xi=3$ and soft-core parameter $a_{0}=0.344$, diagonalization of $U_{\mathrm{CL}}$
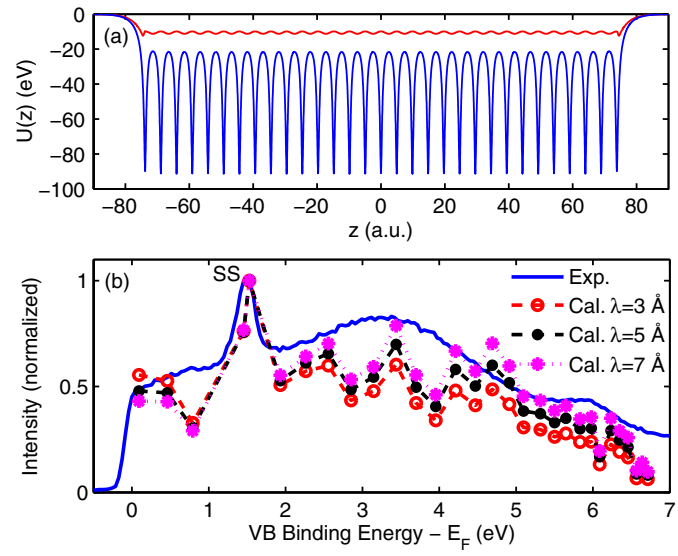

FIG. 1 (color online). (a) Effective VB (top red line) and $2 p$ CL (lower blue line) potentials. (b) PE spectra from the $\mathrm{Mg}(0001)$ VB. Experimental spectrum recorded with $120 \mathrm{eV}$ narrowbandwidth synchrotron radiation as a function of the binding energy relative to the Fermi energy $E_{F}$ [5], and calculated spectra (markers) for $50 \mathrm{fs}, 120 \mathrm{eV}$ XUV pulses and electron MFPs $\lambda_{\mathrm{VB}}=3,5$, and $7 \AA$. The peak at $1.53 \mathrm{eV}$ corresponds to the surface state. All spectra are normalized to their maximum. yields the occupied $\operatorname{Mg}(2 p)$ CL states with a narrow bandwidth of $0.9 \mathrm{eV}$ and binding energies near $-50 \mathrm{eV}$. The energetically highest levels in the narrow $2 p$ CL band have enhanced amplitudes at the slab surfaces.

We use the laser parameters of the experiment [5]. The XUV and NIR electric fields are assumed linearly polarized along the surface normal, approximating grazing incidence of the XUV and NIR pulses. The XUV pulse, $E_{X}(t)=$ $E_{0} e^{-2 \ln 2\left(t / \tau_{X}\right)^{2}} e^{-i\left(\omega_{X} t+\beta t^{2}\right)}$, has the pulse length $\tau_{X}=435$ as (FWHM in intensity), photon energy $\hbar \omega_{X}=118 \mathrm{eV}$, and linear chirp rate $\beta$. The NIR pulse electric field $E_{L}(z, t)=$ $-\partial A_{L}(z, t) / \partial t$ is assumed to be screened at the surface, with a skin depth $\delta_{L}=2 \AA$ [26], and to decay exponentially inside the solid, starting at the image-plane position $z_{\text {im }}=15 a_{s}+3.46$ in the $\operatorname{Mg}(0001)$ Chulkov potential, according to the damping function $f\left(1 / \delta_{L} ; z\right)=\Theta\left(z-z_{i m}\right)+$ $\Theta\left(z_{i m}-z\right) e^{\left(z-z_{i m}\right) / \delta_{L}}$, where $\Theta$ is the unit step function. The NIR pulse vector potential is $A_{L}(z, \hat{t})=A_{0} f\left(1 / \delta_{L} ; z\right)$ $e^{-2 \ln 2\left(\hat{t} / \tau_{L}\right)^{2}} \cos \left(\omega_{L} \hat{t}+\phi\right)$, where $\hat{t}=t-\tau$. $\phi$ is the carrier envelope phase (CEP), $\tau_{L}=5$ fs (FWHM), and $\hbar \omega_{L}=$ $1.5 \mathrm{eV}$. We adjusted $A_{0}$, i.e., the IR-laser peak intensity in our model, to the streaking amplitude in the measured [5] photoemission spectra [cf., Figs. 2(a), 2(b), 2(f), 2(g)

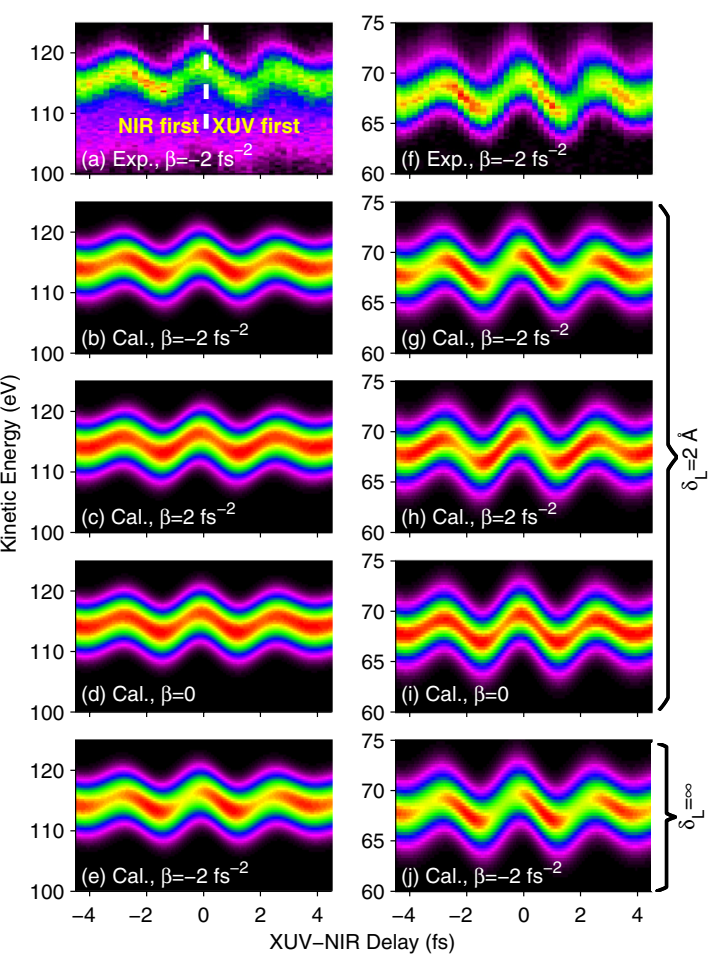

FIG. 2 (color online). Streaking spectrograms of the (a)-(e) VB and (f)-(j) $2 p$ CL band of a $\operatorname{Mg}(0001)$ surface as a function of the XUV-NIR pulse delay $\tau$. The XUV pulse chirp rate is (a),(b),(e), (f),(g),(j) $\beta=-2 \mathrm{fs}^{-2}$, (c),(h) $2 \mathrm{fs}^{-2}$, and (d),(i) 0. (a),(f) Experimental spectra [5]. (b)-(e),(g)-(j) Spectra calculated for a NIR CEP $\varphi=1.22 \pi$ and MFPs (b)-(e) $\lambda=5 \AA$ and (g)-(j) $4 \AA$. The NIR streaking field skin depth is (b)-(d),(g)-(i) $\delta_{L}=2 \AA$ and (e),(j) $\infty$. 
and 3 below]. For delays $\tau>0$ between the centers of the XUV and IR pulse envelopes, the XUV pulse precedes the NIR pulse.

The amplitude for photoemission in the dipole-length gauge and to first order in $E_{X}$ is $[17,19]$

$$
T_{\mathbf{k}_{f}, \mathbf{k}_{i}}(\tau)=-i \int d t E_{X}(t)\left\langle\Psi_{\mathbf{k}_{\mathrm{f}}, \kappa}^{V}(\mathbf{r}, t)|z| \Psi_{\mathbf{k}_{i}}(\mathbf{r}, t)\right\rangle
$$

Assuming translation invariance in the surface $(x-y)$ plane, we write the VB and CL initial states as $\Psi_{\mathbf{k}_{i}}(\mathbf{r}, t)=$ $(2 \pi)^{-1} e^{i \mathbf{k}_{i \|} \mathbf{r}_{\|}} \psi_{i}(z) e^{-i \varepsilon_{i} t}$ with crystal momentum $\mathbf{k}_{i}$ and binding energy $\varepsilon_{i}$. We model the final $\mathrm{PE}$ state in strong-field approximation as a damped Volkov wave function [17] with final momentum $\mathbf{k}_{f}, \Psi_{\mathbf{k}_{f}, \kappa}^{V}(\mathbf{r}, t)=$ $(2 \pi)^{-3 / 2} f(\kappa ; z) e^{i \mathbf{p r}} e^{i \phi_{k_{f z}}(z, t)}$, where the damping factor $\kappa=$ $1 /(2 \lambda)$ is related to the PE MFP $\lambda_{.} \mathbf{p}_{\|}=\mathbf{k}_{f \|}$ is the surface projection of the PE initial kinematic momentum $\mathbf{p}$, and $E=k_{f z}^{2} / 2$ is the final kinetic energy of the PE. Under these assumptions (1) becomes

$$
\begin{aligned}
T_{\mathbf{k}_{f}, \mathbf{k}_{i}}(\tau)= & -i \sqrt{2 \pi} \delta^{(2)}\left(\mathbf{k}_{i \|}-\mathbf{k}_{f \|}\right) \int d t E_{X}(t) e^{-i \varepsilon_{i} t} \\
& \cdot \underbrace{\int d z f(\kappa ; z) e^{-i \phi_{k_{f z}}(z, t-\tau)} e^{-i p_{z}(z, t-\tau) z} z \psi_{i}(z)}_{\text {dipole matrix element }} .
\end{aligned}
$$

We calculate the $z$-dependent Volkov phase $\phi_{k_{f z}}(z, t)=$ $\frac{1}{2} \int_{t}^{\infty} d t^{\prime} p_{z}\left(z, t^{\prime}\right)^{2}$ based on classical photoelectron trajectories $\tilde{z}\left(t^{\prime}\right)$ with initial positions $\tilde{z}(t)=z$ and initial kinematic momenta $p_{z}(z, t)=k_{f z}+\int_{t}^{\infty} d t^{\prime} E_{L}\left[\tilde{z}\left(t^{\prime}\right), t^{\prime}\right]$ at

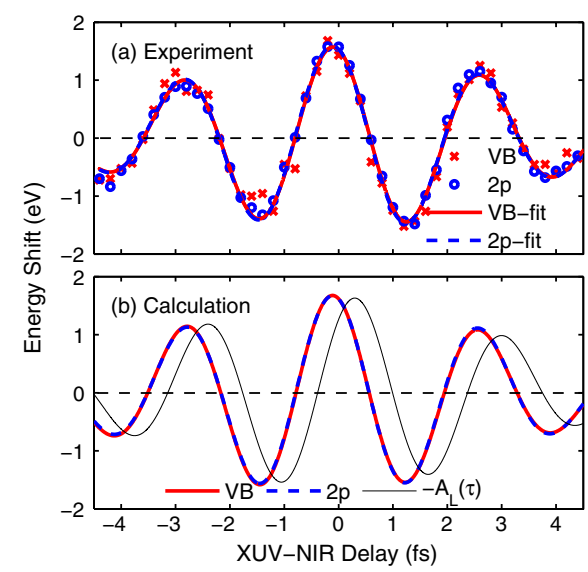

FIG. 3 (color online). Center-of-energy (COE) shifts deduced from (a) measured [5] and (b) calculated streaking traces in Figs. 2(a), 2(f) and 2(b), 2(g), respectively. The curves in (a) are fits to the experimental data (markers). The $2 p$ COE shifts in (b) are multiplied by 1.09 for better comparison. Experimental and calculated results reveal a vanishing relative delay for VB and $2 p$ CL photoemission. The thin black line shows the negative IR vector potential $-A_{L}(\tau)$. the ionization times $t$. The streaked photoemission probability, $P(E, \tau)=\sum_{i}\left|T_{\mathbf{k}_{f}, \mathbf{k}_{i}}(\tau)\right|^{2}$, is obtained by summing over all occupied initial states. The center of energy (COE) as a function of $\tau$ is calculated by averaging $P(E, \tau)$ over the spectral range of a given streaking trace according to $E_{\mathrm{COE}}(\tau)=\int d E E P(E, \tau) / \int d E P(E, \tau)$.

The MFP $\lambda$ describes PE loss due to elastic and inelastic collisions during the propagation inside the solid. It decisively impacts the calculated photoemission probability $[17,20]$, but is difficult to determine accurately [27]. The relative contribution of individual bulk states to the $\mathrm{PE}$ yield in general depends on $\lambda$, while SS PEs are insensitive to variations in $\lambda$ due to their localization at the metalvacuum interface. We calculated the $\mathrm{Mg}(0001) \mathrm{VB}$ XUV $\mathrm{PE}$ spectrum for $50 \mathrm{fs}, 120 \mathrm{eV}$ photon energy pulses and determined the MFP $\lambda_{\mathrm{VB}}$ by fitting the PE spectrum recorded with $120 \mathrm{eV}$ narrow-bandwidth synchrotron radiation [5] [Fig. 1(b)]. We found that the relative contribution to the photoemission yield from states below (above) the SS increases (decreases) with $\lambda$ and obtained the best fit to the experimental VB spectrum for $\lambda_{\mathrm{VB}}=5.0 \AA$. In order to obtain $\Delta \tau_{\mathrm{VB}-2 p}=0$, the MFP for $2 p$ CL PEs must then be adjusted to $\lambda_{2 p}=4.0 \AA$ (cf., Fig. 4 and discussion below). The values we obtained for $\lambda_{\mathrm{VB}}$ and $\lambda_{2 p}$ are in compliance with typical MFP values according to the "universal" MFP curve [28] for electron kinetic energies of $\sim 68 \mathrm{eV}$ and $\sim 115 \mathrm{eV}$ relevant for our investigation. They also agree qualitatively with more recent MFP calculations [27], which predict $\lambda_{\mathrm{VB}}=5.9 \AA$ and $\lambda_{2 p}=4.8 \AA$, and are furthermore in good agreement with the MFP values $\lambda_{\mathrm{VB}}=4.9 \AA$ and $\lambda_{2 p}=3.8 \AA$ extracted from measured XUV PE spectra within a layer-attenuation model [5]. Hence, we use our adjusted values $\lambda_{\mathrm{VB}}=5.0 \AA$ and $\lambda_{2 p}=4.0 \AA$ for calculating streaked PE spectrograms.

Using a quantum-mechanical fitting scheme, Neppl et al. [5] derived the XUV chirp parameter $\beta=-2 \mathrm{fs}^{-2}$ from their measured spectra in Figs. 2(a), 2(f). For the same value of $\beta$ and for $\phi=1.22 \pi$, our calculated streaking spectra in Figs. 2(b), 2(g) agree well with the experimental data and

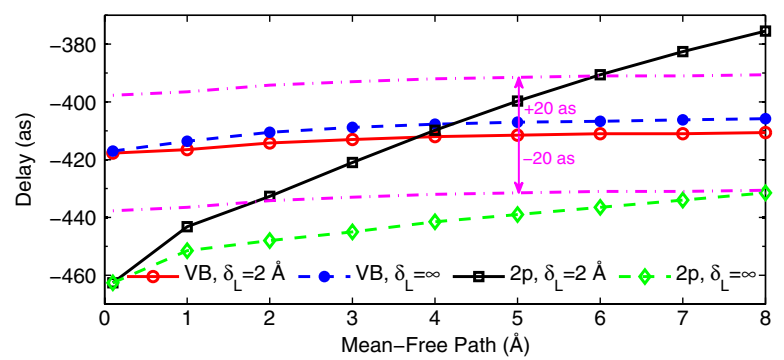

FIG. 4 (color online). Time delay of (a) VB and (b) $2 p$ CL photoemission relative to $-A_{L}(\tau)$ as a function of the MFP for different NIR skin depths $\delta_{L}$. The two magenta dash-dotted lines indicate the experimental standard deviation [5] of the VB emission delay. 
reproduce the $\tau$-dependent change of the shape (i.e., temporal profile) of the PE wave packet. Changes in the temporal PE wave packet profile appear as the conspicuous redistribution of energy-resolved $\mathrm{PE}$ yields inside the streaking traces.

The corresponding COE shifts, derived from the spectra in Figs. 2(a), 2(b), 2(f), 2(g), are shown in Fig. 3. The calculated COE shifts for both streaking traces [Fig. 3(b)] agree well with the experiment [Fig. 3(a)], and our model reproduces the measured vanishing value of $\Delta \tau_{\mathrm{VB}-2 p}$ based on the realistic MFP values $\lambda_{\mathrm{VB}}=5.0 \AA$ and $\lambda_{2 p}=4.0 \AA$. In order to further examine the vanishing relative photoemission delay, we also analyzed contributions to the photoemission yield and delay from individual initial states within the VB and CL band (not shown). The comparison of COEs shows relative emission delays of up to only 6 as for different VB states, but up to 500 as for different $2 p$ CL band states. We traced the large range of $\mathrm{CL}$ delays to different $\mathrm{CL}$ probability densities near the $\mathrm{Mg}(0001)$ surface of different initial states within the narrow CL band. However, contributions from higher $2 p$ CL-band states dominate the $2 p \mathrm{PE}$ yield and determine the $2 p$ emission delay.

The dependence of the VB and CL PE-emission delays on the MFP relative to the phase of the streaking field (NIR vector potential) outside the solid is shown in Fig. 4 for different NIR skin depths $\delta_{L}$. For VB emission, the delay is much less sensitive to changes in the NIR skin depth than for $2 p$ emission. The VB delay increases by less than 10 as for $0.1<\lambda<3 \AA$ and saturates for $\lambda \gtrsim 3 \AA$. For $2 p$ emission, the delay increases almost linearly for $1 \AA<$ $\lambda_{2 p}<8 \AA$ by $\sim 70$ as for $\delta_{L}=2 \AA$ and $\sim 20$ as for $\delta_{L}=\infty$. For $2 p$ emission the efficient screening of the NIR streaking field results in evident delays relative to the unscreened streaking field, consistent with the classical heuristic model [4,5], while for VB emission, our quantum mechanical results are in sharp contrast to those predicted by the classical heuristic model. We ascribe the strikingly different dependence of the VB and $2 p$ photoemission delays on $\delta_{L}$ and the MFPs to the different localization character of VB and $2 p$ CL states [17,21]. More importantly, this difference gives rise to the vanishing delay between $\mathrm{VB}$ and $2 p$ emission, since the unscreened streaking field induces a delay of about 30 as.

The spectral profile of the PE wave packet and its variation as a function of $\tau$ in Fig. 2 are, in general, determined by the spectral profile of the XUV pulse and the dipole matrix element in (2). To assess the influence of $E_{X}(t)$, we compare the spectral profile of streaking traces for different XUV chirp rates $\beta$. For negative $\beta$, the instantaneous XUV frequency decreases with time, so that high (low) spectral components of the PE wave packets are released in the leading (trailing) edge of the XUV pulse [29]. This is best seen by comparing CL photoemission at two consecutive zero-crossings of the vector potential $A_{L}(t)$. For "down-streaking" [corresponding to a negative slope of $A_{L}(t)$, cf., Fig. 3(b)], the higher (lower) PE spectral components will be decelerated (accelerated), giving rise to a narrowed PE energy spectrum with enhanced intensity at the central energy. Conversely, for "up-streaking" [positive slope of $A_{L}(t)$ ], the higher (lower) spectral components will be further accelerated (decelerated), creating a broadened PE spectrum with reduced intensity at the central energy [Figs. 2(g), 2(j)]. For positive $\beta$, the $\tau$-dependent dispersion asymmetry is reversed [Fig. 2(h)]. It disappears at $\beta=0$, as expected [Fig. 2(i)] and is robust against MFP variations (not shown).

Comparing Figs. 2(a)-2(c) and 2(f)-2(h), we find that for finite $\delta_{L}$ the $\tau$-dependent dispersion asymmetry of the $2 p$ CL PEs is much stronger than that of the VB PEs. An unscreened streaking field $\left(\delta_{L}=\infty\right)$ enhances the dispersion asymmetry of the VB PEs [Figs. 2(b), 2(e)], but only slightly modifies the streaking spectra of the $2 p$ PEs [Figs. 2(g), 2(j)]. These differences imply that the spectral profiles of PE wave packets sensitively depend on the screening of the streaking field and the localization character of the initial states in the dipole matrix element.

The above analysis may help to improve the retrieval of XUV pulse parameters from streaked PE spectra in $[4,5]$ with a frequency-resolved optical gating [2,9,30,31] algorithm, especially for VB emission. Neppl et al. [5] reconstructed their measured VB streaking traces from $\mathrm{Mg}$ (0001) surfaces and retrieved the XUV pulse length and chirp assuming a superposition of (localized) Gaussian PE wave packets and an infinite NIR skin depth $\left(\delta_{L}=\infty\right)$. In contrast, our model is based on fully delocalized VB wave functions and best reproduces the experimental data [5] for a finite NIR skin depth. The latter is in agreement with very recent experimental evidence [26]. Since both wave function localization and $\delta_{L}$ strongly influence streaked PE spectra and photoemission time delays, we expect the retrieval algorithm to be significantly more accurate if based on delocalized wave functions and finite skin depths.

In conclusion, we have analyzed the dependence of the $\mathrm{PE}$ dispersion and streaked photoemission time delays for NIR-streaked XUV emission from VB and $2 p$ CL states of a $\operatorname{Mg}(0001)$ surface on the electron MFP, XUV chirp, and NIR skin depth. The large relative photoemission time delay for $\mathrm{W}(110)$ can be related to the different degrees of $\mathrm{CL}$ and VB wave function localizations employing the same (energy-independent) electron MFP for CL and VB emission [16,17] and an infinite NIR skin depth [17]. In contrast, energy-dependent MFPs and finite NIR skin depth must be additionally taken into account in our quantummechanical model to reproduce the measured [5] vanishing relative delay for $\mathrm{Mg}(0001)$ surfaces. VB streaking traces show interesting changes in PE dispersion at a sub-NIRperiod time scale. This XUV-NIR-delay-dependent reshaping of the PE wave packet depends on the modeling of the initial state, propagation effects of PEs in solids, and characteristics (in particular the chirp) of the XUV pulse. 
We thank S. Neppl and R. Ernstorfer for providing the experimental data and R. Kienberger and F. Krausz for valuable discussions. This work was supported by NSF Grant No. PHY 1068752 and the Division of Chemical Sciences, Office of Basic Energy Sciences, Office of Science, U.S. DOE.

[1] M. Hentschel, R. Kienberger, Ch. Spielmann, G. A. Reider, N. Milosevic, T. Brabec, P. Corkum, U. Heinzmann, M. Drescher, and F. Krausz, Nature (London) 414, 509 (2001).

[2] M. Schultze et al., Science 328, 1658 (2010).

[3] K. Klünder et al., Phys. Rev. Lett. 106, 143002 (2011).

[4] A. L. Cavalieri et al., Nature (London) 449, 1029 (2007).

[5] S. Neppl, R. Ernstorfer, E. M. Bothschafter, A. L. Cavalieri, D. Menzel, J. V. Barth, F. Krausz, R. Kienberger, and P. Feulner, Phys. Rev. Lett. 109, 087401 (2012).

[6] C.-H. Zhang and U. Thumm, Phys. Rev. A 82, 043405 (2010).

[7] R. Pazourek, J. Feist, S. Nagele, and J. Burgdörfer, Phys. Rev. Lett. 108, 163001 (2012).

[8] C.-H. Zhang and U. Thumm, Phys. Rev. A 84, 063403 (2011).

[9] F. Quéré, Y. Mairesse, and J. Itatani, J. Mod. Opt. 52, 339 (2005).

[10] V. S. Yakovlev, F. Bammer, and A. Scrinzi, J. Mod. Opt. 52, 395 (2005).

[11] P. B. Corkum and F. Krausz, Nat. Phys. 3, 381 (2007).

[12] J. M. Dahlström, A. L'Huillier, and A. Maquet, J. Phys. B 45, 183001 (2012).

[13] E. E. Krasovskii, Phys. Rev. B 84, 195106 (2011).

[14] C. A. Rios Rubiano, M. S. Gravielle, D. M. Mitnik, and V. M. Silkin, Phys. Rev. A 85, 043422 (2012).
[15] C. Lemell, B. Solleder, K. Tokesi, and J. Burgdörfer, Phys. Rev. A 79, 062901 (2009).

[16] A. K. Kazansky and P. M. Echenique, Phys. Rev. Lett. 102, 177401 (2009).

[17] C.-H. Zhang and U. Thumm, Phys. Rev. Lett. 102, 123601 (2009); Phys. Rev. Lett. 103, 239902(E) (2009).

[18] A. K. Kazansky and P. M. Echenique, Phys. Rev. B 81, 193413 (2010).

[19] J. C. Baggesen and L. B. Madsen, Phys. Rev. A 78, 032903 (2008).

[20] C.-H. Zhang and U. Thumm, Phys. Rev. A 80, 032902 (2009); Phys. Rev. A84, 033401 (2011).

[21] C.-H. Zhang and U. Thumm, Phys. Rev. A 84, 065403 (2011).

[22] E. E. Krasovskii, V. M. Silkin, V. U. Nazarov, P. M. Echenique, and E. V. Chulkov, Phys. Rev. B 82, 125102 (2010).

[23] A. G. Borisov, D. Sanchez-Portal, A. K. Kazansky, and P. M. Echenique, Phys. Rev. B 87, 121110(R) (2013).

[24] E. V. Chulkov, V. M. Silkin, and P. M. Echenique, Surf. Sci. 437, 330 (1999).

[25] F. Schiller, M. Heber, V. D. P. Servedio, and C. Laubschat, Phys. Rev. B 70, 125106 (2004).

[26] R. Kienberger and F. Krausz (private communication).

[27] S. Tanuma, C. J. Powell, and D. R. Penn, Surf. Interface Anal. 43, 689 (2011).

[28] A. Zangwill, Physics at Surfaces (Cambridge University Press, Cambridge, England, 1988).

[29] S. Neppl, Ph.D. Thesis, Technische Universität München (2012).

[30] Y. Mairesse and F. Quéré, Phys. Rev. A 71, 011401 (2005).

[31] J. Gagnon, E. Goulielmakis, and V. S. Yakovlev, Appl. Phys. B 92, 25 (2008). 